HISTORIA DE LA MEDICINA

\title{
La epidemia de viruela en la Alcaldía Mayor de Tegucigalpa año 1815
}

\author{
The smallpox epidemic in the Mayoralty of Tegucigalpa year 1815
}

\author{
José Manuel Cardona Amaya
}

Máster en Historia Social y Cultura; Departamento de Historia, Facultad de Ciencias Sociales, Universidad Nacional Autónoma de Honduras, Tegucigalpa.

RESUMEN. En 1815 una epidemia de viruela ingresó a la Alcaldía Mayor de Tegucigalpa que, a pesar de los esfuerzos de las autoridades por esparcir la vacuna, causó estragos en la población indígena. Al mismo tiempo, en la época lluviosa de ese año, las lluvias fueron menores a lo acostumbrado, provocando el fracaso de las plantaciones, lo que llevó a una escasez de granos y a una hambruna generalizada en el territorio. En este escenario, el Alcalde Mayor Simón Gutiérrez se vio obligado a cobrar el tributo de los pueblos indígenas, maniobrando políticamente la situación para evitar alguna revuelta de la población. La epidemia de viruela y la hambruna ejemplifican la aplicación de política sanitaria en la Honduras colonial, destacando las maneras en que las acciones individuales de los funcionarios eran un ejercicio de administración de la vida de los habitantes de la provincia. De manera similar, en 2020 los intereses políticos y económicos han interferido en la prevención efectiva contra el COVID-19.

Palabras Clave: Epidemiología, Historia, Honduras, Organización y administración, Políticas.
Recibido: 16-05-2020 Aceptado para publicación 11-12-2020

Dirección para correspondencia: Master José Manuel Cardona Amaya

Correo electrónico: jmcardona@unah.edu.hn

Declaración de relaciones y actividades financieras y no financieras y conflictos de interés: ninguno.

DOl: https://doi.org/10.5377/rmh.v88i2.11497

\section{INTRODUCCIÓN}

Cinco años antes de la independencia de Honduras, la Alcaldía Mayor de Tegucigalpa sufrió una de sus crisis más graves: en 1815, una epidemia de viruela ingresó desde Guatemala al territorio, diezmando la población indígena. En la época lluviosa de ese mismo año, las lluvias fueron menores de lo acostumbrado, conduciendo esto a una escasez de alimentos y a una hambruna generalizada en 1816. Estos dos eventos se verían agravados por la restitución del pago de los tributos que habían sido abolidos con la constitución de 1812. Con la población enferma y hambrienta, las autoridades españolas de la Alcaldía Mayor de Tegucigalpa debieron emprender la difícil tarea de cobrar los tributos sin provocar una insurrección por el descontento de la población.

La epidemia de viruela de 1815 es un fenómeno conocido por la historiografía nacional, aunque poco estudiado, no existiendo hasta el momento un estudio a profundidad sobre el suceso. Entre los textos que mencionan este evento, Linda Newson $^{1}$ específica que en esta plaga murió un $18 \%$ de los indios tributarios de Choluteca; mientras que Luis Taracena ${ }^{2}$ señala a esta enfermedad como uno de los factores determinantes en la disminución de la población de Yuscarán. En ocasiones se menciona este fenómeno como la plaga de 1816, años en que la enfermedad alcanzó su ápice en la Alcaldía Mayor; sin embargo, se trató de un brote único de enfermedad que inició en Guatemala en los primeros meses de 1814 y luego descendió a la Alcaldía Mayor de Tegucigalpa a finales de ese año.

El presente artículo se desarrolló con el propósito de explorar y analizar los eventos de la plaga de viruela de 1815 y la hambruna de 1816, para comprender los efectos que estos tuvieron en la población de la Alcaldía Mayor de Tegucigalpa. Entre estos se entrecruza el drama del cobro de los tributos del primer tercio del año de 1816, una situación que causó tensión entre los poblados indígenas afectados por ambos males y las autoridades españolas. El enfoque principal de este análisis está dirigido a las acciones de los funcionarios públicos frente a la enfermedad y la hambruna. La situación era en extremo compleja: aunque el pago del tributo era compulsorio de parte de los indios, también era obligación de los funcionarios españoles cobrarlo, obviando el estado de crisis. Por lo que la comprensión del ejercicio de la administración de salud se vuelve fundamen- 
tal para entender el alcance de la vacuna, la distribución de los alimentos y el cobro de los tributos. Los datos para este análisis han sido extraídos del Fondo Colonial del Archivo Nacional de Honduras, utilizándose un total de trece documentos, con un rango de fechas de 1815 a 1818. Esta información ha sido analizada cronológicamente y pormenorizadamente.

\section{LA EPIDEMIA DE VIRUELA DE 1815}

En 1814, desde Guatemala, se anunciaba la venida de una nueva plaga. A mediados de ese año, los niños de la ciudad de Guatemala fueron afectados por una enfermedad descrita como de toses y, suponiendo las autoridades del gobierno superior que esta peste se extendería por el resto del territorio centroamericano, se envió instrucciones a Tegucigalpa de cómo lidiar con ese mal. ${ }^{3}$ Para noviembre, en la Villa de Tegucigalpa ya se hacían planes de cómo llevar la vacuna de la viruela a esa ciudad, en prevención de que brotara una nueva plaga. Las sospechas de los funcionarios de Tegucigalpa se confirmaron el 26 de febrero de 1815, al arribar noticias que en Guatemala la enfermedad se encontraba ya esparcida por la mayoría de poblaciones. Esta enfermedad llegó el mismo año a la Alcaldía Mayor de Tegucigalpa, habiendo un par de registros de los estragos que causó. En Texiguat se reportó que todas las familias se encontraban postradas en cama y que, añadido a esto, una escasez de alimentos los había asolado; ${ }^{4}$ en Linaca se informó que la plaga de viruela los había afectado tanto en 1815 como en $1816 .^{5}$

En atención a la plaga, las autoridades de la Alcaldía Mayor de Tegucigalpa enviaron dos jóvenes a traer el fluido de la vacuna de la viruela a Comayagua y, de no estar disponible allí, a cualquier ciudad cercana en que estuviese. El expediente en que se consigna esta orden también aporta una descripción de la convocatoria que se haría en la Villa de Tegucigalpa para vacunar a su población:

Se publique pr. [sic] Bando en el primer día de la próxima Pasqua [sic] p.a [sic] q.e [sic] concurran a una de las casas del Alc.e [sic] Mayor Ynt.o [sic], del Padre Cura y Vic.o [sic], ô del vocal Regid.r [sic] D. Manuel Antonio Vásquez, todos los que no estubieren [sic] vacunados a que se les administre esta operación p.r [sic] los vacunadores instruidos Secretarios D. Joaq.n [sic] San Martin, D. Vicente Coronel, y Martin Suniga [sic], a quienes deberán pagar un real p.r [sic] cada vacunado. ${ }^{6}$

Que los habitantes de la Villa hayan sido requeridos ir a las casas de los funcionarios era algo normal, ya que por el pequeño tamaño de la población se esperaba que todos conocieran la ubicación de estos edificios. Existe un documento sumamente valioso en el que se consignan las personas vacunadas en la Villa de Tegucigalpa. ${ }^{7}$ Esta lista fue realizada por Martín Zúñiga, uno de los vacunadores y remitida a las autoridades de la Villa el 21 de mayo de 1816. Según el documento, hasta esa fecha se habían vacunado 76 españoles y 1020 pardos, lo que otorga un total de 1096 vacunados. Sin embargo, en su suma del total, el vacunador consigna la cifra de 1276 vacunados, lo que puede explicarse por un error de suma o por la existencia de un informe previo que dé constancia de las 180 personas restantes. Entre los españoles vacunados en esa ocasión se encuentra Francisco Morazán, el futuro presidente de la Federación Centroamericana, además de su futura esposa María Josefa Lastiri.

En contraste a la Villa de Tegucigalpa, los pueblos de indios sufrieron grandes penas por la plaga de viruela. Una carta del Capitán Francisco Antonio Márquez dirigida al Alcalde Mayor Simón Gutiérrez, aporta evidencia para argumentar que entre los indios había cierta resistencia hacia la vacuna. El Capitán comunicó lo siguiente:

La peste de viruelas de este pueblo continua con toda fuersa [sic] la que no he podido cortar como se ha hecho con otros lugares a pesar de los esfuersos [sic] q.e [sic] he hecho para propagar la vacuna; pues estos yndios [sic] han sido ingratos a Dios, en no hacer aprecio de este admirable remedio que nos descubrió y al Rey que ha dado las mas exâtas [sic] providencias p.a [sic] que nos aprovechemos de él. ${ }^{8}$

Los datos recabados permiten hacer la siguiente apreciación: en la Alcaldía Mayor de Tegucigalpa, la aplicación de la vacuna contra la viruela fue más exitosa en los centros urbanos de españoles que en los poblados rurales de indios. Esto queda de manifiesto en dos hechos: primero, el informe anteriormente citado demuestra que, para mediados de 1816, la mayoría de la población de la Villa de Tegucigalpa se encontraba ya vacunada; segundo, que, en ese mismo año, varios pueblos de indios fueron incapaces de pagar sus tributos, solicitando esperas al gobierno colonial.

A la plaga de viruela se sumó que en la época lluviosa de ese año las lluvias fueron pocas, provocando una escasez generalizada de granos en la Alcaldía Mayor de Tegucigalpa. Era este un hecho catastrófico, ya que la plaga de viruela había incapacitado a gran parte de la población indígena, la cual ahora debía de lidiar con la falta de comida. En una carta dirigida al Alcalde Mayor Simón Gutiérrez, el cura de Alubarén describió las muertes causadas por ambos males, especificando que la mayoría de indios había muerto por la hambruna y no por la viruela:

A penas, de haber yo, como párroco, ya con alim.tos [sic] ya con medicinas, se me ha muerto la m.or [sic] parte de hambre, haciéndomelo assi [sic] decir, ver con mis ojos, q.e [sic] la convaleciencia [sic] fue con la xicama [sic], cirguela [sic] o jocote, de tal suerte, q.e [sic] hasta ahora, hay varios, no vuelven en sí. ${ }^{9}$

Se tiene constancia de la hambruna en las siguientes poblaciones: en Aguanqueterique, Alubarén, Reytoca, Curaren y Tatumbla, ${ }^{10}$ Linaca, ${ }^{11}$ Tizcagua, $^{12}$ Ojojona ${ }^{13}$ y Texiguat. ${ }^{14}$ Esta distribución geográfica de la hambruna permite hacer la afirmación de que se trataba de un problema generalizado en toda la Alcaldía Mayor de Tegucigalpa y de mayor impacto en las poblaciones indígenas.

Las autoridades de la Alcaldía Mayor de Tegucigalpa se encontraban ante una situación delicada, pues en marzo de ese año se debía pagar el tercio de San Juan, un tributo que había sido restablecido mediante cédula de 1814. El cobro de este tributo confrontaba directamente al poder español en Tegucigalpa con los pueblos de indios de su jurisdicción, los cuales además 
de ser azotados por la viruela y la hambruna ahora debían de pagar un impuesto obligatorio. Si bien es cierto que el Alcalde Mayor de Tegucigalpa quiso alegar ante el superior gobierno que él no sabía cuánto cobrar por haberse quedado la matrícula de los pueblos en la Intendencia de Comayagua, los fiscales de la Real Hacienda ratificaron la obligatoriedad del tributo.

Los documentos dan testimonio de cómo los pueblos indios fueron incapaces de rendir este tributo por los dos males que sufrían. En el siguiente pasaje, las autoridades indígenas del pueblo de Texiguat comunican al Alcalde Mayor Simón Gutiérrez que no podían continuar cobrando el tributo a sus pobladores, tanto por la viruela como por la "necesidad" -la hambruna-:

Noticiamos a Vmd [sic] p.r [sic] quanto [sic] al padecer de la común peste que esta en este mi pueblo muriendo mucha gente tributario, juntam.te [sic] la necesidad que estamos padeciendo gragravemente [sic] mortal p.r [sic] no poder Buscar, la providencia de mantencion [sic] para sustentar los enfermos p.r q.e [sic] se ta [sic] mirando que los que lebantan [sic] de la enfermedad de la peste según la necesidad tan grande que esta a lebantado [sic] de la cama y si an [sic] muerto p.r [sic] fatalidad, de la necesidad y p. [sic] lo tanto patentisamos [sic] a Ntrô S.r [sic] Alcalde mayor, que ya no allamos [sic] como cobrar los derechos de tributos. ${ }^{15}$

Otros pueblos de indios dirigieron cartas similares al Alcalde Mayor. Por ejemplo, los principales de Langue solicitaron que se les extendiera el plazo del pago hasta agosto a lo que el Alcalde Mayor contestó no tener competencia para conceder esta extensión pero que esperaba que ellos cumplieran su palabra. Una intercesión similar fue hecha por Florencio Valerio de parte del pueblo de Tizcagua, solicitando una extensión del pago hasta el 15 de agosto. Mientras tanto, los indios de Aguanqueterique, Alubarén, Reytoca, Curaren y Tatumbla, alegaron ante el Alcalde Mayor que se debía de realizar una nueva tasación del tributo, ya que la plaga de viruela y la hambruna habían matado a varios tributarios de sus pueblos, conduciendo a que los sobrevivientes se vieran cargados con el pago de sus cuentas; en ese informe se menciona también que el pueblo de Lauterique había hecho una solicitud similar.

Lo cierto era que el Alcalde Mayor no tenía la competencia de gobierno para conceder prórroga en el pago de los tributos, responsabilidad que recaía en los oficiales reales del Rey. Ante la gran cantidad de pueblos indígenas afectados por la viruela y la hambruna, el 5 de agosto de ese año, el Alcalde Mayor decidió enviar un informe al Superintendente General del Reino, para que este determinase si el atraso del pago era justificado. Este documento es especialmente valioso, porque ofrece la perspectiva del funcionario superior del territorio ante las calamidades que habían asolado su jurisdicción. Simón Gutiérrez, el Alcalde Mayor, describió los grandes problemas que afectaban el pago del tributo indígena. Mediante sus palabras introductorias, el Alcalde Mayor buscaba descargarse de la culpa del atraso del tributo ante el Superintendente General, demostrándole que él había tomado las precauciones necesarias, repartiendo la vacunación por los poblados de su jurisdicción y marchado hasta otras provincias para proveer de alimentos a sus habitantes. El plan del Alcalde Mayor era el mismo que el de los indios: solicitar que se replantease el cobro de los tributos porque las tasaciones habían sido hechas antes de que la viruela y hambruna matasen a una parte significante de la población. El Real Tribunal de Cuentas contestó al Alcalde Mayor el 26 de agosto, comunicándole que el cobro debía de hacerse con las matrículas de los pueblos estimadas en 1811.

Aunque su solicitud ante el superior gobierno fue fallida, la petición del Alcalde Mayor logró ganarles tiempo a los indios para reunir el dinero de los tributos. No se reportan conflictos generados por el cobro de este tributo, por lo que ha de suponerse que a finales de ese año de 1816 fue posible cobrarlo 0 alcanzar una resolución con la Real Hacienda.

A pesar de haberse introducido en 1816, hay evidencia que sugiere que la vacuna contra la viruela tuvo una aceptación lenta en la población de la Alcaldía Mayor de Tegucigalpa. En un escrito de 1819, Santiago Boezo comunicó al Alcalde Mayor que tenía problemas para hacer llegar la vacuna a los poblados del partido de Ojojona, ya que no había persona capacitada para vacunar y los ladinos no acudían a los llamados para adoptar esta medida.

\section{CONCLUSIONES}

La Alcaldía Mayor de Tegucigalpa enfrentó una de sus mayores crisis en 1816, afectada por una epidemia de viruela y una hambruna, aunado a la obligación de pagar el tributo real. Gran parte de la catástrofe fue evitable, teniéndose noticias del brote de la epidemia desde 1814 y circulando por Centroamérica la vacuna desde varios años atrás. Sin embargo, los funcionarios españoles de la Alcaldía Mayor de Tegucigalpa no se lanzaron a actuar hasta que la enfermedad ya había arribado a su jurisdicción. A partir de 1815, una política sanitaria torpe y excluyente llevó a la agudización de la crisis. Se importó la vacuna, pero se colocó en lugares relacionados con el poder, en los centros urbanos de españoles, con el objetivo de inocular primero a la población privilegiada del sistema de castas. Fue hasta meses después que la vacuna partió con rumbo a las poblaciones indígenas, de mano de los tenientes de partido y sacerdotes, agentes externos a las comunidades que levantaban ciertas sospechas por la naturaleza de esa cura novel. Al no poder vacunarse la mayoría de la población indígena, la enfermedad los afectó con impunidad. Esto precipitó una tragedia mayor: ya que una sequía había afectado las plantaciones y la mayoría de indios no podía levantarse de sus camas por estar postrados por la viruela, la hambruna mató a una parte significativa de la población indígena. Ante esta crisis sin precedentes, los funcionarios españoles de la Alcaldía Mayor actuaron para preservar la vida de los habitantes, buscando alimentos en otras jurisdicciones, acelerando la propagación de la vacuna e interviniendo ante las autoridades superiores para la prorrogación del tributo obligatorio. A pesar de las acciones loables del Alcalde Mayor ante la crisis, se debe de concluir que esta pudo reducirse mediante pre- 
venciones al momento de enterarse de la enfermedad y con una política sanitaria inclusiva que protegiese a los más vulnerables.

La relación entre la salud pública y la política es fundamental para comprender por qué en Honduras ciertas epidemias han causado tantos estragos. De la misma manera como en 1815 la inoculación contra la viruela se vio entorpecida por las acciones de los funcionarios políticos, en el año 2020 los intereses políticos y económicos han interferido en la preven- ción efectiva contra el COVID-19. En un editorial reciente, HI Codina ha apuntado como en Honduras, las élites económicas y políticas han velado por su propia salud durante la pandemia de COVID-19, en detrimento de las clases sociales con menor acceso a recursos. ${ }^{16}$ Lo anterior demuestra que, en la Honduras del siglo XXI, se siguen cometiendo los mismos errores que en el siglo XIX, anteponiéndose los fines de ciertos individuos a la salud de la población en general.

\section{REFERENCIAS}

1. Newson L. El costo de la conquista. Tegucigalpa: Guaymuras; 1992.

2. Taracena L. Ilusión minera y poder político: la Alcaldía Mayor de Tegucigalpa siglo XVIII. Tegucigalpa: Guaymuras; 1996.

3. Borjas P. (Funcionario del Imperio Español). Carta a: los cabildos seculares de los pueblos de Ojojona, Aguanqueterique, Goascorán, Nacaome, Choluteca, El Corpus, Texiguat, Yuscarán y San Antonio (Alcaldía Mayor de Tegucigalpa). 23 de noviembre de 1991. 4 páginas. Localizada en: Archivo Nacional de Honduras, Tegucigalpa, Honduras; Caja 113, documento 3652.

4. Mendoza U. (Funcionario del Imperio Español). Carta a: Simón Gutiérrez (Alcalde Mayor de Tegucigalpa). 29 de junio de 1816. 4 páginas. Localizada en: Archivo Nacional de Honduras, Tegucigalpa, Honduras; Caja 119, documento 3854 .

5. Lagos R. (Cura y rector de la villa de Choluteca). Carta a: Simón Gutiérrez (Alcalde Mayor de Tegucigalpa). 20 de enero de 1818. 2 páginas. Localizada en: Archivo Nacional de Honduras, Tegucigalpa, Honduras; Caja 127, documento 4194

6. Espinoza J. (Funcionario del Imperio Español). Carta a: los cabildos seculares de Ojojona, Aguanqueterique, Goascorán, Nacaome, Pespire, La Venta, Sabanagrande y Santa Ana (Alcaldía Mayor de Tegucigalpa). 27 de febrero de 1815. 8 páginas. Localizada en: Archivo Nacional de Honduras, Tegucigalpa, Honduras; Caja 114, documento 3680.

7. Zúñiga M. (Encargado de la vacunación en Tegucigalpa). Carta a: Simón Gutiérrez (Alcalde Mayor de Tegucigalpa). 21 de mayo de 1816. 12 páginas. Localizada en: Archivo Nacional de Honduras, Tegucigalpa, Honduras; Caja 119, documento 3837.

8. Márquez A. (Teniente de Alcalde Mayor). Carta a: Simón Gutiérrez (Alcalde Mayor de Tegucigalpa). 13 de julio de 1816. 4 páginas. Localizada en: Archivo Nacional de Honduras, Tegucigalpa, Honduras; Caja 120, documento 3868 .

9. Alvarado J. (Teniente de cura de Texiguat). Carta a: Simón Gutiérrez (Alcalde Mayor de Tegucigalpa). 12 de julio de 1816. 4 páginas. Localizada en: Archivo Nacional de Honduras, Tegucigalpa, Honduras; Caja 120, documento 3866

10. Alvarado J. (Teniente de cura de Texiguat). Carta a: Simón Gutiérrez (Alcalde Mayor de Tegucigalpa). 5 de agosto de 1816. 4 páginas. Localizada en: Archivo Nacional de Honduras, Tegucigalpa, Honduras; Caja 120, documento 3892.

11. Lagos R. (Cura y rector de la villa de Choluteca). Carta a: Simón Gutiérrez (Alcalde Mayor de Tegucigalpa). 22 de julio de 1816. 4 páginas. Localizada en: Archivo Nacional de Honduras, Tegucigalpa, Honduras; Caja 120, documento 3880

12. Valerio F. (Teniente de Alcalde Mayor). Carta a: Simón Gutiérrez (Alcalde Mayor de Tegucigalpa). 20 de julio de 1816. 4 páginas. Localizada en: Archivo Nacional de Honduras, Tegucigalpa, Honduras; Caja 120, documento 3876

13. Castejón M. (Teniente de Alcalde Mayor). Carta a: Simón Gutiérrez (Alcalde Mayor de Tegucigalpa). 15 de julio de 1816. 4 páginas. Localizada en: Archivo Nacional de Honduras, Tegucigalpa, Honduras; Caja 120, documento 3870.

14. Arrollave A. (Funcionario del Imperio Español). Carta a: Simón Gutiérrez (Alcalde Mayor de Tegucigalpa). 3 de septiembre de 1816. 8 páginas. Localizada en: Archivo Nacional de Honduras, Tegucigalpa, Honduras; Caja 120, documento 3906 .

15. Mendoza U. (Funcionario el Imperio Español). Carta a: Simón Gutiérrez (Alcalde Mayor de Tegucigalpa). 19 de junio de 1816. 4 páginas. Localizada en: Archivo Nacional de Honduras, Tegucigalpa, Honduras; Caja 119, documento 3851

16. Codina HI. COVID-19 en Honduras. Rev Méd Hondur. 2020;88(1): 6.

ABSTRACT. In 1815, a smallpox epidemic entered the Mayoralty of Tegucigalpa, which, despite the authorities' efforts to spread the vaccine, wreaked havoc on the indigenous population. At the same time, in the winter of that year, the rains were less than usual, causing the failure of plantations, which led to a shortage of grain and widespread famine in the territory. In this scenario, Mayor Simón Gutiérrez was forced to collect tribute from indigenous peoples, politically maneuvering the situation to avoid any revolt by the population. The smallpox epidemic and famine exemplify the application of sanitary politics in colonial Honduras, highlighting the ways in which the individual actions of the officials were an exercise in the administration of the life of the inhabitants of the province. Similarly, by 2020 political and economic interests have interfered with effective prevention against COVID-19.

Keywords: Epidemiology, History, Honduras, Organization and History, Policies. 